BNL-99879-2012-CP

\title{
Optimization of NSLS-II Blade X-ray Beam Position Monitors: from Photoemission type to Diamond Detector
}

\author{
P. Ilinski \\ Submitted to Journal of Physics: Conference Series
}

March 2013

Photon Sciences Directorate

Brookhaven National Laboratory

\section{U.S. Department of Energy DOE - Office of Science}

Notice: This manuscript has been authored by employees of Brookhaven Science Associates, LLC under Contract No. DE-AC02-98CH10886 with the U.S. Department of Energy. The publisher by accepting the manuscript for publication acknowledges that the United States Government retains a non-exclusive, paid-up, irrevocable, world-wide license to publish or reproduce the published form of this manuscript, or allow others to do so, for United States Government purposes.

This preprint is intended for publication in a journal or proceedings. Since changes may be made before publication, it may not be cited or reproduced without the author's permission. 


\section{DISCLAIMER}

This report was prepared as an account of work sponsored by an agency of the United States Government. Neither the United States Government nor any agency thereof, nor any of their employees, nor any of their contractors, subcontractors, or their employees, makes any warranty, express or implied, or assumes any legal liability or responsibility for the accuracy, completeness, or any third party's use or the results of such use of any information, apparatus, product, or process disclosed, or represents that its use would not infringe privately owned rights. Reference herein to any specific commercial product, process, or service by trade name, trademark, manufacturer, or otherwise, does not necessarily constitute or imply its endorsement, recommendation, or favoring by the United States Government or any agency thereof or its contractors or subcontractors. The views and opinions of authors expressed herein do not necessarily state or reflect those of the United States Government or any agency thereof. 


\title{
Optimization of NSLS-II Blade X-ray Beam Position Monitors: from Photoemission type to Diamond Detector
}

\author{
P Ilinski ${ }^{1}$ \\ Brookhaven National Laboratory, Upton NY, USA \\ E-mail: pilinski@bnl.gov
}

\begin{abstract}
Optimization of blade type X-ray Beam Position Monitors (XBPM) was performed for NSLS-II undulator IVU20. Blade material, configuration and operation principle was analyzed to improve XBPM performance. Optimization is based on calculation of the XBPM signal spatial distribution. Along with standard photoemission blades, Diamond Detector Blade (DDB) was analyzed as XBPM signal source. Analyses revealed, that Diamond Detector Blade XBPM would allow overcoming drawbacks of the photoemission type XBPMs.
\end{abstract}

\section{Introduction}

Photoemission blade X-ray BPMs [1] are standard for most synchrotron radiation facilities. The photoemission XBPMs are non-invasive and can provide high spatial resolution, but they are vulnerable to the background radiation from dipoles and focusing optics due to their high sensitivity to the lower energy photons. Performance of the photoemission type XBPM was analyzed to optimize geometry and configuration of photoemission blades. Optimization is based on calculations of the XBPM signal spatial distribution. An alternative type of a Diamond Detector Blade XBPM [2] was analyzed and compared to the photoemission XBPM.

\section{Undulator Radiation Source}

Optimization of the XBPM was performed for the NSLS-II undulator IVU20. The power density spatial distribution at the minimum undulator gap, corresponding to $\mathrm{K}=1.8$, and at $500 \mathrm{~mA}$ storage ring current is shown at figure 1 . The power density distribution specifies the XBPM blades high heat load conditions and defines how far the blades should be placed away from the axis of radiation. Blades need to withstand the heat load and should be mechanically stable since blade deformation can be interpreted as the undulator beam motion.

Another important characteristics of undulator radiation for XBPM operation is the spectral flux angular dependence. The flux spectral density of IVU20 at various locations from the axis of undulator radiation at $10 \mathrm{~m}$ is presented in figure 2 which shows that hard X-rays from high undulator harmonics are radiated at large off-axis angles.

\section{Photoemission Blade XBPM}

Operation of the photoemission XBPM is based on photoemission of electrons from a blade. Tungsten is often used as a blade material for undulator XBPMs due to mechanical properties. The resulting photoemission XBPM signal is convolution of the undulator spectral density and the total electron yield of the blade. Convolution for a portion of the undulator spectra reveals signal distribution for particular undulator harmonic. The signal spatial distributions of tungsten blade for the first and second undulator harmonics of IVU20, $\mathrm{K}=1.8$, at $10 \mathrm{~m}$ are shown at figures 3 and 4 . The signal generated by second undulator harmonic is more intense compare to the signal due to the first

1 To whom any correspondence should be addressed. 
harmonic and is localized closer to the axis of undulator radiation. The total signal spatial distribution of tungsten blade XBPM, which includes all undulator harmonics, is presented at figure 5 .

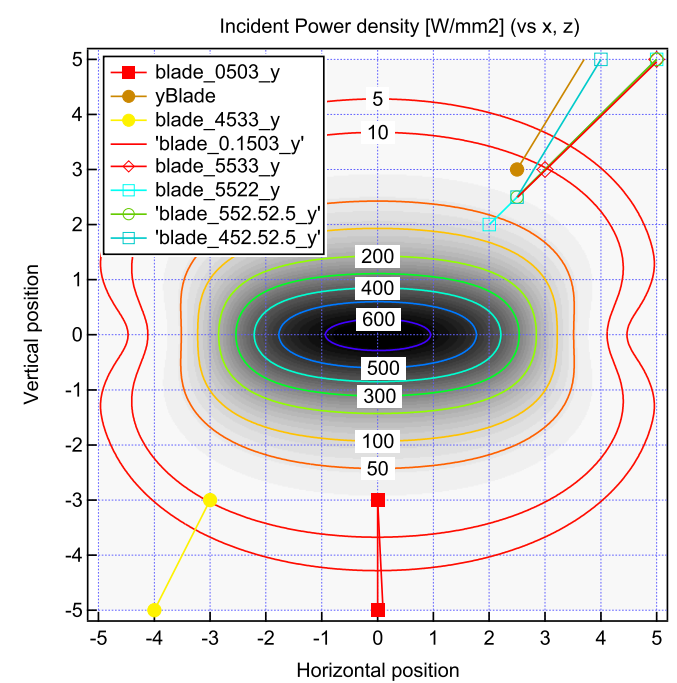

Figure 1. Power Density spatial distribution of undulator radiation, IVU20, $\mathrm{K}=1.8,10 \mathrm{~m}$.

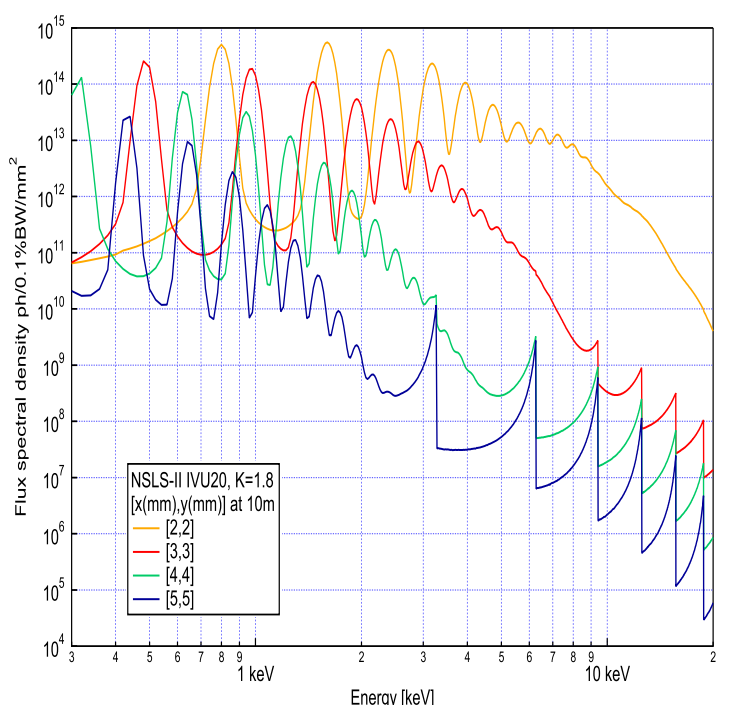

Figure 2. Flux Spectral Density angular dependance IVU20, $\mathrm{K}=1.8,10 \mathrm{~m}$ at various $[\mathrm{mm}$, $\mathrm{mm}]$ locations from the axis of radiation.

For blades shown at figure 5 the signal level per tungsten blade is reaching few hundreds of microamperes at 10m distance for IVU20 at $500 \mathrm{~mA}$ current and $\mathrm{K}=1.8$, reducing to tens of micro-amperes for $\mathrm{K}=0.5$. The signal level will increase if blades are extended towards the axis of radiation, but this is limited by the high heat load conditions.

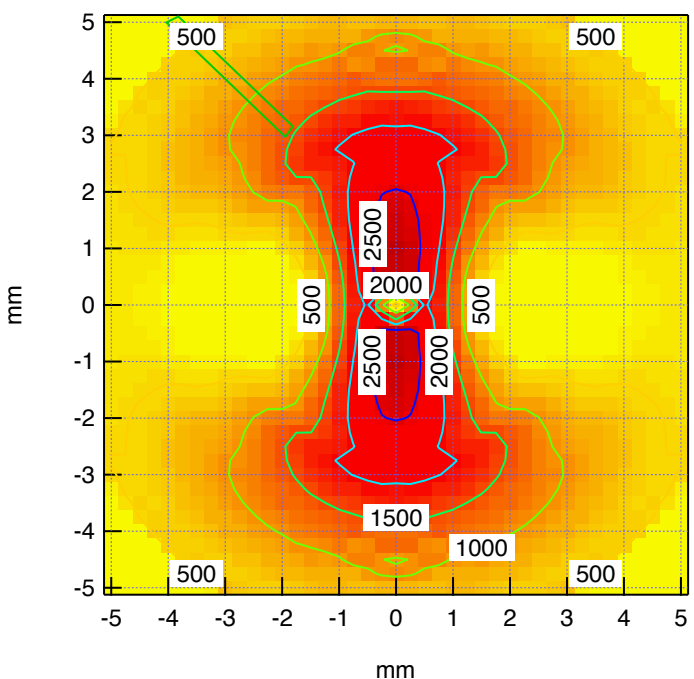

Figure 3. The signal spatial distribution for tungsten blade XBPM for the first undulator harmonic, IVU20, $\mathrm{K}=1.8,10 \mathrm{~m}$.

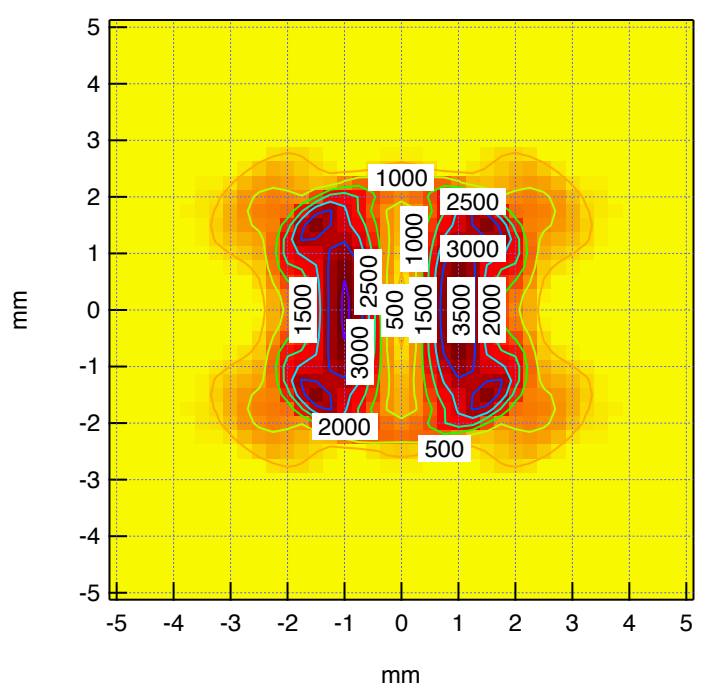

Figure 4. The signal spatial distribution for tungsten blade XBPM for second undulator harmonic, IVU20, $\mathrm{K}=1.8,10 \mathrm{~m}$.

Calibration curves, calculated as $(\mathrm{S} 1-\mathrm{S} 2) /(\mathrm{S} 1+\mathrm{S} 2)$, where $\mathrm{S} 1$ and $\mathrm{S} 2$ are signals from the opposite blades, are presented at figure 6 for different configurations of blades indicated in figure 5 . As can be 
seen, the calibration curves do not differ significantly when beam deviation is smaller then $0.5 \mathrm{~mm}$. Therefore, the sensitivity of the photoemission XBPM cannot be improved substantially by optimization of the blade configuration after the blade geometry was defined to satisfy the high heat load conditions.

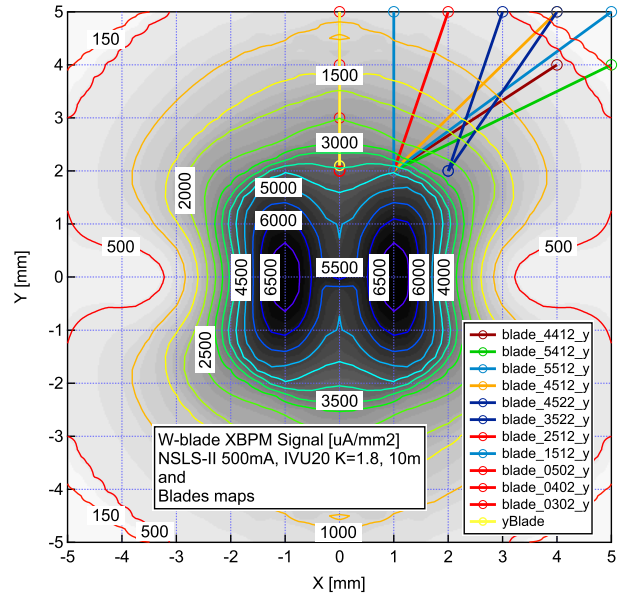

Figure 5. Tungsten blade XBPM. The total signal spatial distribution, IVU20, $\mathrm{K}=1.8$, $10 \mathrm{~m}$.

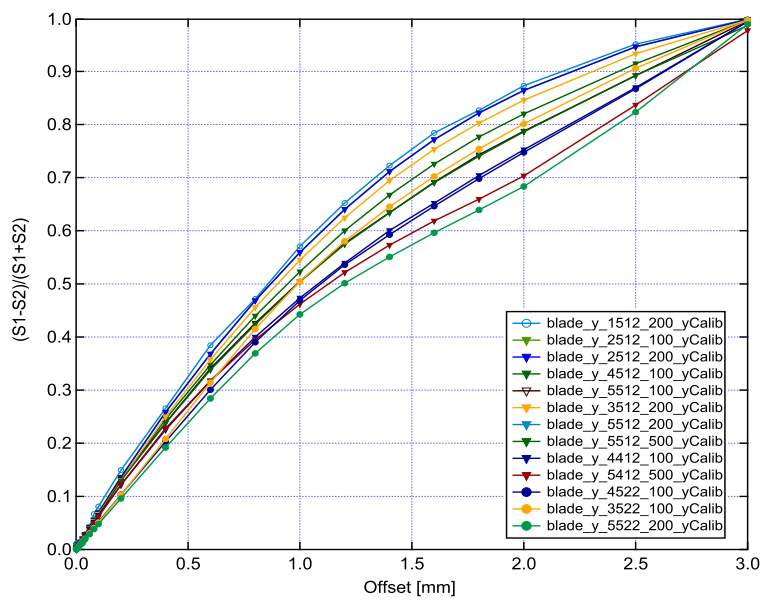

Figure 6. Tungsten blade XBPM. The calibration curves for various blades configurations.

\section{Diamond Detector Blade XBPM}

The major deficiencies of the photoemission XBPM are sensitivity to the lower energy photons and dependence on the condition of photoemission surface. Those deficiencies can be overcome by changing the photoemission blade to the Diamond Detector Blade. The DDB XBPM was introduced by H. Aoyagi [2], the layout of the DDB is shown at figure 7. The DDB is positioned along the radiation axis similar to the photoemission blade. Charge carriers generated when photon is absorbed in the diamond detector drift to opposite side electrodes when bias voltage is applied. The signal generated in the diamond detector is proportional to the number and energy of absorbed photons through conversion factor of $\sim 13 \mathrm{eV} / \mathrm{e}-\mathrm{h}$ [3]. This provides an intrinsic discrimination for the lower energy photons. Further discrimination can be achieved by not collecting charge carriers generated by lower energy photons at the front of the DDB by offsetting the electrodes from the edge of the DDB, figure 7. This is similar to an introduction of an X-ray filter in front of the DDB.

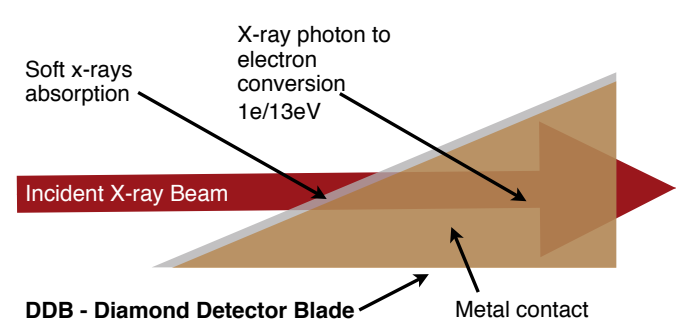

Figure 7. Layout of the diamond detector blade of the DDB XBPM.

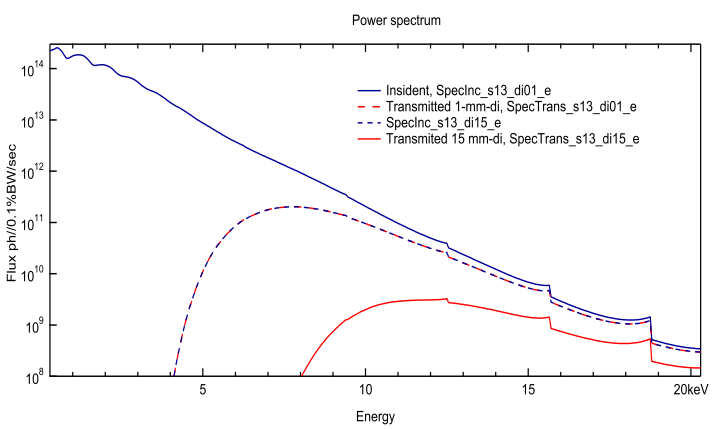

Figure 8. Integrated Spectral Flux, IVU20, $\mathrm{K}=1.8$. Incident undulator radiation transmitted after $1 \mathrm{~mm}$ thick diamond, and transmitted after $16 \mathrm{~mm}$ thick diamond. 
The integrated spectral flux of undulator radiation from IVU20 at $\mathrm{K}=1.8$ is shown at figure 8 along with the integrated spectral flux transmitted after $1 \mathrm{~mm}$ and $16 \mathrm{~mm}$ thick diamonds. As can be seen, photons with energies below $4 \mathrm{keV}$ are absorbed in the $1 \mathrm{~mm}$ thick diamond filter. Varying thickness of the X-ray filter by changing the geometry of side electrodes will make possible to discriminate the lower energy photons background radiation and to control the DDB signal level. Optimization of the side electrodes geometry will also allow control of DDB characteristics such as capacitance.

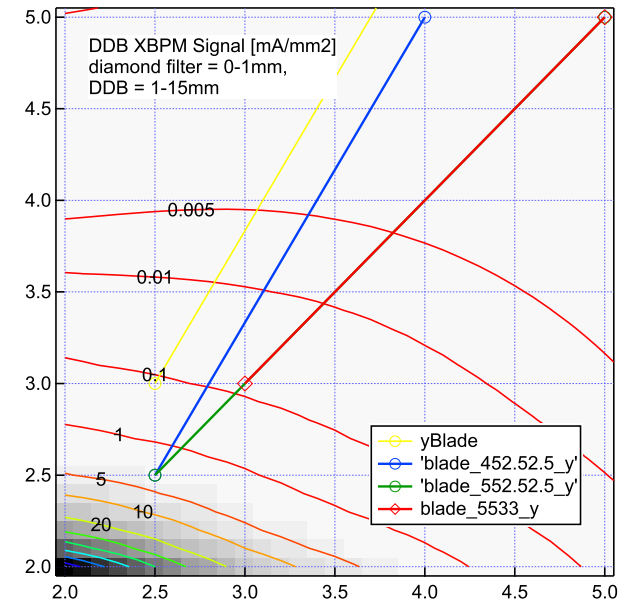

Figure 9. The signal spatial distribution of the DDB XBPM, IVU20, $\mathrm{K}=1.8,10 \mathrm{~m}, 1 \mathrm{~mm}$ thick diamond $\mathrm{X}$-ray filter.

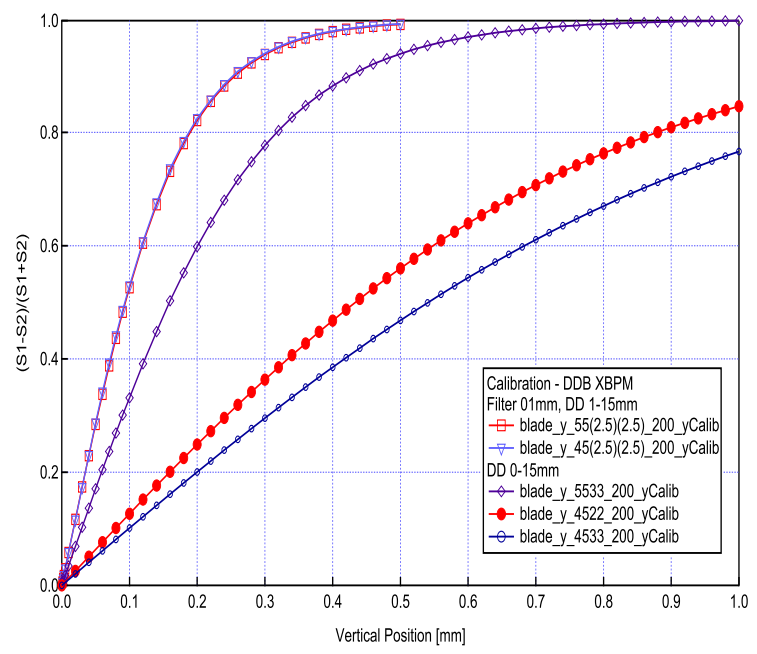

Figure 10. The DDB XBPM calibration curves with and without diamond X-ray filter for various blade configurations.

The spatial distribution of the DDB XBPM signal reflects the spatial distribution of the incident radiation power density. The DDB signal is reaching level of few hundreds of milli-amperes for IVU20, $\mathrm{K}=1.8$, at $500 \mathrm{~mA}$ storage ring current. When a $1 \mathrm{~mm}$ thick diamond $\mathrm{X}$-ray filter is introduced in front of the DDB, the signal spatial distribution becomes narrower, figure 9 , the signal level drops to hundreds of micro-amperes. The calibration curves of the DDB XBPM with and without diamond Xray filter are presented at figure 10 for various blade configurations. The sensitivity of the DDB XBPM without X-ray filter is two times higher compare to the tungsten blade XBPM, figure 6 . The sensitivity of the DDB XBPM with X-ray filter depends on the blade configuration and is 6-8 times higher compare to the tungsten blade XBPM.

\section{Conclusion}

A noninvasive type of the white beam Diamond Detector Blade XBPM was analyzed and compared to the photoemission XBPM. The choice of the Diamond Detector Blade instead of the photoemission blade as XBPM signal source allows discrimination of the lower energy background photons. The discrimination is achieved due to proportionality of the DDB signal to the energy of absorbed photon, and by additional X-ray filtering through modification of the DDB side electrodes.

\section{References}

[1] Johnson E D and Oversluizen T 1989 Compact flux beam position monitor Rev. Sci. Instrum. 60 1947

[2] Aoyagi H, Kudo T, Tanida H, and Kitamura H 2004 New configuration of photoconductive type diamond detector head for X-ray beam position monitors AIP Conf. Proc. 705933

[3] Pomorski $\mathrm{M}$ et al 2005 Characterization of single crystal CVD diamond particle detectors for hadron physics experiments Phys. Stat. Sol. (a) 2022199 